\title{
Studies on Supported Employment for People with Intellectual Disabilities
}

\author{
Hu Chenyu \\ Nanjing Foreign Language School, Nanjing, China
}

Email address:

huchenyu514@126.com

To cite this article:

Hu Chenyu. Studies on Supported Employment for People with Intellectual Disabilities. Humanities and Social Sciences.

Vol. 5, No. 4, 2017, pp. 145-149. doi: 10.11648/j.hss.20170504.13

Received: June 21, 2017; Accepted: July 10, 2017; Published: July 19, 2017

\begin{abstract}
This article, based on the case management model from Nanjing BOAI Center, analyzes the achievements of supported employment (SE) and the difficulties, including lack of funds, shortage of job coaches, a few positions for people with intellectual disabilities, etc. In this paper, the purpose or purported significance of the study is clearly stated. The author introduces the research background clearly and some related works are cited. The methods section provides sufficient information. In addition, the references of this paper are the latest. But some suggestions should be paid attention to: improving employment policies for people with intellectual disabilities, strengthening the training of the job coaches, and creating more work opportunities.
\end{abstract}

Keywords: People with Intellectual Disabilities, Supported Employment, Support Strategy

\section{Introduction}

Employment rate symbolizes the level of economic development and social civilization of a country. As a difficult group among the disabled persons, people with intellectual disabilities cannot find suitable jobs because of their physiological conditions, cultural views, social environment and the level of industrial development. According to the results of the second national disability sampling survey in 2006, 2.97 million people with intellectual disabilities are employed, and 4.7 million people with intellectual disabilities are unemployed. The employment rate for the disabled is about $38.7 \%$, while that for people with intellectual disabilities is lower to $10 \%$ [1]. Thus it can be seen that the problems of SE still need further study.

At present, the main employment model of people with intellectual disabilities is sheltered employment in China. This is a centralized employment model directed by the government. It is a protected, intermittent, temporary and transitional form of employment. The main problem of the sheltered employment model is the separation between the workplace and the society, in which the disabled, especially those with intellectual disabilities, are excluded from the normal social life. That is not conducive for the disabled to integrate into the society and to enjoy the same life as others.
Moreover, with the development of Chinese Economic Reform and Open up and intensified mark competition, sheltered employment is not appropriate now.

SE is a model of employment for people with disabilities (mainly intellectual disabilities), adhering to the "placement ahead of training" principle. That is to help the disabled to acquire "real work" in workshops under, considering their ability, interest and the right to select jobs. Research has shown that the primary achievement of SE is to increase the income of people with intellectual disabilities, promote social integration, and greatly improve their quality of life [2]. Kramer, McIntyre, and Blacher (2003) studied 188 people with moderate or severe disabilities, and found that those who did "real work" had higher quality of life compared with those who did traditional sheltered work. SE has been widely accepted in the world and has become a new inclusive model.

SE provides a new direction for the employment for people with intellectual disabilities. A few of organizations in China have made active exploration in this field. It is meaningful to study their practice.

\section{Research Progress}

In view of the existing literature, there are a few special studies of the employment for people with intellectual 
disabilities in China. Lu Tianqing, evaluated the employment status of people with intellectual disabilities in Shanghai by means of questionnaire and interview, summed up the reasons for the employment difficulties of people with intellectual disabilities and put forward suggestions for the construction of employment support network for people with intellectual disabilities from the macro, meso and micro perspectives [3]. Li Jin, with her analysis of traditional intellectual disabilities sheltered employment, introduced "SE" concept and designed the implementation process of SE, in order to provide a new model of advanced intellectual disability employment in China and improve the quality of employment service for people with intellectual disabilities [4]. Zhou Ziyu analyzed the international excellent experience and gave the localization strategy and pointed out that sustained support and follow-up counseling should be provided in the SE services program, which will provide more rationalized advice for the job coaches' work process [5]. Zhuo Caiqin et al. summed up the SE of Hui Ling in Guangzhou, analyzed the problems in SE, and eventually constructed the SE model for people with intellectual disabilities [6].

Based on the relevant domestic literature, some people have introduced theories about SE and proposed suggestions about its framework; however, there are few empirical researches of non-governmental organizations' practice to explore SE. In this article, literature researches and interviews are adopted to sum up experience and analyze existing difficulties in the practice of SE by Nanjing BOAI Center, and then suggestions about promoting SE are presented, from the perspectives of the government, enterprises, organizations, people with intellectual disabilities and their families.

\section{Present Situation of SE}

\subsection{Introduction of the SE Model}

Adopted by the federal government, The Developmental Disabilities Act of 1984 explains SE as follows: The Senate amendments would define "supported employment" to mean paid employment for persons for whom competitive employment at or above the minimum wage is unlikely and who, because of their disabilities, need intensive, ongoing support in a work setting, including settings in which nonhandicapped persons are employed. The support includes any activity needed to sustain paid work including supervision, training and transportation [7].

SE consists of three basic elements: payment, integrated work settings, and follow-up services. It has following four steps: (a) assess the characteristics and needs of people with disabilities and arrange them suitable positions; (b) give direct or indirect training and guidance combined with the support system in the workplace provided by the job coach at site; (c) reduce support from job coaches when the work performance of the supporters meets requirements; (d) provide follow-up support as needed. SE overcomes the lack of learning ability among people with intellectual disabilities and has good practicability.

\subsection{Current Situation of China's SE}

In 90s, Professor Xu Jiacheng of Beijing Union University College of Special Education visited Taiwan for the first time. Professor $\mathrm{Xu}$ studied new changes in the definition of intellectual disability, especially concept of "supported" (supported life, supported education, SE), and then introduced the concept to mainland [8]. Around 2000, some institutions in Chongqing and Beijing began to experiment SE and achieved certain results, but not influential.

Since 2012, the International Labour Organization, China Disabled Persons' Federation (CDPF), association of friends and relatives, CDPF Employment Guidance Center and other organizations have cooperatively promoted the "SE model". Beijing, Shandong, Hunan, Guangxi, Jilin, Shenzhen, Dalian and other seven provinces and cities have been selected as the pilot. At present, there are more than 200 cases of successful employment [9]. In June 2015, the Opinions on the development of auxiliary employment for people with intellectual disabilities, issued by CDPF and other eight ministries, clarifies:...strengthen the training of job coaches, provide SE services, and help the mentally and physically handicapped persons who are qualified integrate into the labor market and achieve employment.

Although China's public figures and government are actively exploring the SE, SE is still in its infancy due to social environment and other factors. The government departments have been familiar with sheltered employment, while unfamiliar with SE.

\section{SE Practice from Nanjing BOAI Center}

Although there are no relevant policies and regulations for SE in Nanjing, some non-governmental organizations have taken action. Nanjing BOAI Center, founded in 2010, is a non-governmental organization dedicated to serving adults with intellectual disabilities and their families. Its main functions include day care, rehabilitation training, skills training and sheltered employment services for people with intellectual disabilities. In 2013, BOAI Center applied for the venture philanthropy project [10] offered by Nanjing Xuanwu district government in order to set up a case management model for the employment of intellectual disabilities. "Case management" model is an one to one individual service model that job coaches help individuals to get employed. This project, launched from June 2013 to May 2014, received more than 100 thousand yuan by the Xuanwu District Civil Affairs Department in Nanjing. It takes SE as the core and guidance and has started to explore SE for people with intellectual disabilities in Nanjing.

At the same time, BOAI Center had participated the training and research program of SE for Chinese intellectual and developmental disabilities [11] which carried out by the Institute of special education of Beijing Union University and commissioned by the International Labor Organization. This project aimed to establish an SE system for people with 
intellectual disabilities by means of the evaluation and support strategy, "SE" training for job coaches and services for employment, based on social support theory.

\subsection{Project Operation Process}

The SE model adopted in the project can be summarized as "3-6-8". "3" refers to three basic steps of the SE, including explore, training and support. "6" refers to six processes of this project: job founding, workplace analysis, job matching, centralized vocational training, workplace support, ongoing support. "8" refers to the continuous steps of this project, including:

(a) Investigation. By means of home visits, telephone interviews, parental counseling and other forms, this project investigates the employment needs of 118 families that meet project requirements in Xiaolingwei Street and sets up files for individuals who have the employment intention.

(b) New work opportunities. The project successfully cooperated with three enterprises to create more work opportunities in Nanjing.

(c) Assessment. Scales, such as Personal Outcome Scale, provided by the International Labour Organization for job coaches training, are adopted to assess the capabilities and needs of supported employees.

(d) Vocational training. The project cooperated with the Labor Department of Xuanwu District CDPF and offered housework, handcraft, computer and paper cutting and other training programs, according to the needs of supported employees.

(e) Job training. With the help of Human Resource departments of enterprises, the project carried out the job training, including theoretical study and practical operation.

(f) Internship. The job coach selects potential individuals to practice in a caring workshop.

(g) Job matching. After internship, the job coach re-assesses the individuals' capability and selects the suitable ones for the job.

(h) Ongoing support. During individuals' working period, job coach provides them with necessary help, analyzes successful cases of employment and re-visits the employed and cooperative enterprises.

\subsection{Achievement of the Project}

At the end of the project, 11 people with intellectual disabilities signed formal labor contracts with employers. 63 of the 96 people with intellectual disabilities participating in the vocational training were qualified. 35 intellectual disabilities participated in job training. According to the follow-up, 9 of these 11 persons with intellectual disabilities remained employed, and 2 of them did not due to the changes in family and work environment and other reasons.

The project had positive impacts for people with intellectual disabilities, their families, employers and societies. After the successful employment of people with intellectual disabilities, their families living conditions had been greatly improved, and their parents felt less worried about their disabled offspring. Employers said that people with intellectual disabilities were more responsible than ordinary people, and that they would create more positions for them. The above results show that it is feasible and effective to carry out SE by case management model for people with intellectual disabilities. SE can promote the employment of more people with intellectual disabilities and a more inclusive society.

\subsection{Problems}

Nanjing BOAI Center were unable to continue to carry out the case management model to support people with intellectual disabilities people after the project was over. The main reasons for its inconsistency were lack of funds, lack of professional job coaches, a few jobs provided, and lack of family support.

\subsubsection{Lack of Funds}

Case management model needs amounts of money in operation. This project applied for special funds from Nanjing Xuanwu District CDPF in the name of venture philanthropy project. In process of the project, Nanjing Xuanwu District CDPF also provided training places and expert resources. But the project also needed to pay for training materials, transportation, accommodation, and job coaches allowance, etc. Special funds merely sustained the necessary cost of the project. Moreover, the special funds were one-off, so that the government did not continue to provide support unless a new project successfully applied for funds. In accordance with the Law on the Protection of the Rights and Interests of Persons with Disabilities and the Employment Regulations for People with Intellectual Disabilities, the government may collect employment security funds for the disabled from employers who do not employ persons with disabilities. However, because there is no corresponding policy support, these funds are difficult to invest non-governmental organizations to support the disabled employment projects. Meanwhile, due to the lack of society's recognition, it is hard for organizations to obtain funds from the society. Consequently, the lack of funds has been the main reason for the inconsistency of the project.

\subsubsection{Lack of Professional Job Coaches}

Job coaches play a key role in the process of the SE. They help the disabled with job hunting, workplace training and long-term follow-ups; they also work as a link among enterprises, the disabled and their families. These jobs require job coaches to have all kinds of professional knowledge. At the beginning of the project, there was only one professional coach who was familiar with the concept and process of SE. In the course of the project, she took two courses organized by the Institute of special education of Beijing Union University and contributed to the effective promotion of the project; however, she left her job afterwards. Since the project did not continue, and there was no standardized training mechanism and qualification verified mechanism for job coaches in China, Nanjing BOAI Center did not train more job coaches 
afterwards. There was no job coach who continuously tracked the employment situation with intellectually disabled people after the project was over. When working, people with intellectual disabilities often encounter two problems: one is that they have suffered from negative opinions and thus refuse to accept positive advice; the other is that they reject or do not know how to ask for help. Insufficient follow-ups failed to keep the supported employees employed in long term. Therefore, job coaches need do more to help them build better interpersonal relationship.

\subsubsection{A Few Jobs Provided}

Generally, there are a few employers who are willing to employ people with intellectual disabilities, and jobs provided for people with intellectual disabilities are limited. Although a few regional CDPFs have sponsored special recruitment for people with intellectual disabilities, most employers refuse to employ them due to the assumption that they are unsuitable for the job. In order to promote the employment of the disabled, the Employment Regulations for People with Disabilities has clarified measures, including the minimum proportion of the employment of people with disabilities in enterprises, the employment security funds for the disabled, and the responsibility of governments at or above the county level to create jobs suitable for the disabled. However, many employers think that the payment of employment security funds is less than the cost of employing the corresponding number of people with intellectual disabilities; they would rather pay the employment security than hire people with disabilities. The insufficient social acceptance, lack of professional job coaches, and ineffective government's policies concerning employment of people with intellectual disabilities have led to the shortage of job opportunities, which becomes the key factor that hinders the development of SE.

\subsubsection{Lack of Family Support}

Families with the intellectual disabled are not optimistic about their intellectual disabled family members' employment in the future. Guardians worry that the high mobility of job coaches will lead to the unstable employment of people with intellectual disabilities; that organizations can not find insufficient reliable job information; and that the intellectually disabled may suffer discrimination and unfair treatment. In order to prevent the intellectually disabled from being harmed in an open social environment, their families tend to let them live in a closed environment. This is no good for the promotion of the supported development.

\section{Proposals for SE}

The difficulties faced by the Nanjing BOAI Center in the process of SE practice are highly representative and reflect the plight of SE to some extent. The implementation of SE cannot only rely on the resources of non-governmental organizations. It further needs extensive support from the government, enterprises and society.

\subsection{Perfect Policies and Enhance Support for Non-governmental Organizations That Implement SE}

Non-governmental organizations may play a important role in the practice of SE, as can be seen from the case above. Nevertheless, they do not have sufficient funds and human resources to keep SE in operation; they need to be supported by the government. Therefore, the government should hurry to carry out supportive policies to facilitate the work of non-governmental organizations. For example, government may further improve the Employment Regulations for Disabled Persons to stipulate that the employment security for disabled persons can be used to support non-governmental organizations to practice SE; and that a certain amount of compensation will be granted for non-governmental organizations based on their SE results, in order to encourage more civil forces to participate in the SE services. Only when the government cooperates with civil forces can they create a better supportive social environment and promote the normal employment of people with intellectual disabilities.

\subsection{Cultivate Job Coaches by the Lead of the Government}

Job coach plays a vital role in SE. They introduce the SE concept, have professional skills, organize and coordinate resources, and ensure successful employments. The government should include the expenditure of training job coaches in the budget system; establish and improve the training mechanism of job coaches; absorb abroad experience and set up standardized job coaches' qualification examinations; specify the duty of job coaches and raise the salary and welfare level appropriately to make the job more attractive and competitive. In this way, enough professional job coaches will engage in SE.

\subsection{Create More Work Opportunities}

\subsubsection{Provide Suitable Jobs for People with Intellectual Disabilities by Learning from the Successsful Models}

To help people with intellectual disabilities get jobs, some practitioners, including associations of parents, long-term cooperative corporations, and caring people in the society, created some employment models according to intellectually disabled person's own condition. These models can be popularized. One example is a model called "XIHANER Washing Car Center". This center emerged in the effort of the parents raising money and hiring car washing professionals to help the intellectually disabled learn how to wash the cars. This model is a combination of centralized employment and decentralized employment: the workplace is open, and the employment of people with intellectual disabilities is relatively concentrated. Both the supported employees and customers can communicate at any time, which helps the supported employees participate in social life. Another example is the "Gump restaurant." Employers provide work places, and job coaches give specific training for the supported employees. The supported employees may have the job after they have fit in the position. Moreover, "HANLEER-Spring Sunshine-Tea Story" (a tea shop's name) is a third example 
that promotes the employment of the intellectually disabled in the community. It has four sponsors: (a) local government provides places, water and electricity; (b) social organization, especially parents of people with intellectual disabilities, provides funds; (c) philanthropy enterprises provide free "Tea Story" brand and technical guidance; (d) professional team is responsible for the management.

\subsubsection{Enhance Community Service and Arouse the Sense of Belonging in the Mind of People with Intellectual Disabilities}

The study found that people with intellectual disabilities who show high levels of basic living skills in the community can live and work independently [12]. Local CDPF, communities and the disabled persons' associations can associate with universities, media, enterprises and community social workers to shoulder the responsibilities of recommending and transferring employment and create nearby and simple jobs for the people with intellectual disabilities, such as working in steamed bread room, distributing newspapers, and etc. Let them get employed by the help of acquaintance, especially family members.

\subsection{Increasing the Publicity of SE}

In fact, discrimination against people with intellectual disabilities is still prevalent in the community. Many people still hold the belief that the intellectually disabled cannot work. However, it has proved that the intellectually disabled are capable for competitive work if appropriate supportive services are provided. Therefore, the society shall promote the social recognition and integration of people with intellectual disabilities and change the conventional views of the intellectually disabled that they are unproductive by reporting successful cases of intellectual disabled persons' employment via television, internet, public service ads, non-commercial performances, etc. Eventually, people with intellectual disabilities not only have rights, but also live with the dignity. Families with people with intellectual disabilities should change their focus from family care to supports for independent employment, and from reliance on subsidy to creation of values. They may create a supportive family atmosphere for people with intellectual disabilities to grow up independently and go out for job. Enterprises may take an open attitude to allow the intellectually disabled get employed and set working standards which are especially designed according to each supported employee's job setting, working hours, and welfare protection.

\section{Conclusion}

It is the achievements of SE that people with intellectual disabilities can get rid of isolated and enclosed space, live independently, and integrate themselves into society. SE has received more attention in China. The more people dedicate themselves to SE, the more people with intellectual disabilities will return to the society and participate in social life equally, which makes the society more harmonious.

\section{References}

[1] 2006 Second National Disability Sampling Survey, Major Data Bulletin (No. 1), 2006.

[2] Daniel E. Steere, et al. Growing up: Transition to Adult Life for Students with Disabilities. Boston, MA: Pearson Allyn and Bacon, 2007.

[3] Lu Tianqing. Study on the Employment of People with Intellectual Disabilities in Shanghai [D], East China Normal University, May 2008.

[4] Li Jin. Thoughts on Carrying out Supported Employment for People with Intellectual Disabilities in China [J], Journal of Jixi University, November 2011.

[5] Zhou Ziyu, National Experience and Localization Strategies for Supported Employment of People with Intellectual Disabilities [J], Modern Special Education, March 2016.

[6] Zhuo Caiqin, et al. Construction of Supported Employment Model for People with Intellectual Disabilities -- Based on the Practical Research of "Guangzhou Hui Ling", http://www.capidr.org.cn/news192.html, June 13, 2013.

[7] Rusch F R, Hughes C. Overview of Supported Employment [J]. Journal of Applied Behavior Analysis, 1989, (22):351-363.

[8] Xu Jiacheng, A New Evolution in the Definition of Mental Retardation -- A New Trend Based on "Function", "Support" and "Quality of Life" [J]. Special Education in China, April 2003.

[9] Zhang Baolin, The Employment of Mentally Retarded People and Supported Employment Model-speech at the Twelfth Beijing Forum

http://blog.sina.com.cn/s/blog 439a914e0102wc9c.html, November 7, 2015.

[10] Cong Ming, A Case Management Model for the Sustainability of Employment Programs for People with Intellectual Disabilities - Take A Project in Nanjing for Example, [D], December 23, 2016.

[11] Program for Training and Research on Supported Employment for Intellectual and Developmental Disabilities in China, http://www.capidr.org.cn/news156.html, August 1, 2013.

[12] Zhou Haibin, ILO and International Support for People with Intellectual Disabilities in China, http://www.capidr.org.cn/news988.html, September 1, 2013. 